\title{
Article
}

\section{The Importance of Clinical Examination under General Anesthesia: Improving Parametrial Assessment in Cervical Cancer Patients}

\author{
Paulina Sodeikat ${ }^{1}$, Massimiliano Lia ${ }^{1}$, Mireille Martin ${ }^{2}$, Lars-Christian Horn ${ }^{3}$, Michael Höckel ${ }^{1}$, \\ Bahriye Aktas ${ }^{1}\left[\right.$ and Benjamin Wolf ${ }^{1, *}$ (i)
}

1 Department of Gynecology, Leipzig University Medical Center, D-04103 Leipzig, Germany; paulina.sodeikat@web.de (P.S.); massimiliano.lia@medizin.uni-leipzig.de (M.L.); michael.hoeckel@medizin.uni-leipzig.de (M.H.); bahriye.aktas@medizin.uni-leipzig.de (B.A.)

2 Department of Diagnostic and Interventional Radiology, Leipzig University Medical Center, D-04103 Leipzig, Germany; mireille.martin@medizin.uni-leipzig.de

3 Division of Gynecologic, Breast, and Perinatal Pathology, Leipzig University Medical Center, D-04103 Leipzig, Germany; lars-christian.horn@medizin.uni-leipzig.de

* Correspondence: benjamin.wolf@medizin.uni-leipzig.de; Tel.: +49-341-97-23459

check for

updates

Citation: Sodeikat, P.; Lia, M.; Martin, M.; Horn, L.-C.; Höckel, M.; Aktas, B.; Wolf, B. The Importance of Clinical Examination under General Anesthesia: Improving Parametrial Assessment in Cervical Cancer Patients. Cancers 2021, 13, 2961. https://doi.org/10.3390/ cancers13122961

Academic Editor: Ruud L. M. Bekkers

Received: 5 May 2021

Accepted: 9 June 2021

Published: 13 June 2021

Publisher's Note: MDPI stays neutral with regard to jurisdictional claims in published maps and institutional affiliations.

Copyright: (c) 2021 by the authors. Licensee MDPI, Basel, Switzerland. This article is an open access article distributed under the terms and conditions of the Creative Commons Attribution (CC BY) license (https:/ / creativecommons.org/licenses/by/ $4.0 /)$.
Simple Summary: In most cases, the treatment strategy (radiation or surgery) in cervical cancer patients depends on whether the parametrium shows tumor involvement. Traditionally, clinical pelvic examination under general anesthesia (EUA) has been used to determine whether tumor spread into the parametrium is present. During the recent decade, however, magnetic resonance imaging (MRI) has been increasingly used to determine whether parametrial tumor extension is present, and several studies have indicated that MRI might be superior to EUA. In this study, we demonstrate that EUA still plays an important role in pre-therapeutic evaluation of cervical cancer patients, and that display of MR images in the operating room (augmented EUA) achieves superior results in predicting parametrial tumor spread when comparted to MRI alone, especially in larger tumors. Best predictive results were observed in cases when radiologists and gynecological oncologists agreed on parametrial status, highlighting the importance of interdisciplinary patient assessment.

Abstract: Background: Parametrial tumor involvement is an important prognostic factor in cervical cancer and is used to guide management. Here, we investigate the diagnostic value of clinical examination under general anesthesia (EUA) and magnetic resonance imaging (MRI) in determining parametrial tumor spread. Methods: Post-operative pathological findings of 400 patients with primary cervical cancer were compared to the respective MRI data and the results from EUA. The gynecological oncologist had access to the MR images during clinical assessment (augmented EUA, aEUA). Results: Pathologically proven parametrial tumor invasion was present in 165 (41\%) patients. aEUA exhibited a higher accuracy than MRI alone (83\% vs. $76 \%$; McNemar's odds ratio [OR] = 2.0, $95 \%$ CI 1.25-3.27, $p=0.003$ ). Although accuracy was not affected by tumor size in aEUA, MRI was associated with a lower accuracy in tumors $\geq 2.5 \mathrm{~cm}$ (OR for a correct diagnosis compared to smaller tumors $0.22, p<0.001)$. There was also a decrease in specificity when evaluating parametrial invasion by MRI in tumors $\geq 2.5 \mathrm{~cm}$ in diameter $(p<0.0001)$ compared to smaller tumors $(<2.5 \mathrm{~cm})$. Body mass index had no influence on performance of either method. Conclusions: aEUA has the potential to increase the diagnostic accuracy of MRI in determining parametrial tumor involvement in cervical cancer patients.

Keywords: cervical cancer; magnetic resonance imaging; examination under anesthesia; accuracy; sensitivity; specificity; predictive value 


\section{Introduction}

In 2019, the Fédération Internationale de Gynécologie et d'Obstétrique (FIGO) released a revised cervical cancer staging system which calls for the incorporation of imaging modalities for the first time [1]. Studies suggest that evaluation of cervical cancer using magnetic resonance imaging (MRI) is superior to evaluation by clinical examination in earlier cancer stages (IB1-IIA2) [2-6]. However, the roles of MRI and clinical examination in advanced stages, characterized by tumor extension beyond the uterine cervix, remain unclear. Especially tumor spread into the laterally abutting supporting tissues, the so called parametria (stage IIB), is of importance as it is usually considered to be a contraindication for surgical treatment. In a systematic literature review of studies published between 2012 and 2018, Woo et al. [5] found that sensitivity for detection of parametrial infiltration (PMI) by MRI was $76 \%$ and specificity was $94 \%$. These numbers are concordant with two meta analyses which included studies conducted within the past 30 years $[4,7]$. Of note, only four out of the 14 studies included in these analyses involved patients with cancer staged IIB or higher. In contrast, sensitivity for the detection of parametrial infiltration by clinical examination varies between $29 \%$ and $66 \%$ [4,5,8-11]. In the reported data, specificities for detecting parametrial invasion by clinical examination versus magnetic resonance imaging range from $81 \%$ to $99 \%$ for clinical examination and from $63 \%$ to $99 \%$ for MRI $[8,9,12-14]$

Most studies investigating parametrial tumor involvement are limited by the circumstance that comprehensive post-operative histology for locally advanced cases is not available as patients with presumed parametrial infiltration undergo primary chemoradiotherapy at most institutions, in accordance with current national and international guidelines [15,16].

Since parametrial invasion (PMI) is an established factor governing treatment decisions [15] and prognosis [17,18], more information regarding the value of MR imaging and clinical examination to detect parametrial cancer spread is needed. During the past two decades, we conducted the Leipzig School Mesometrial Resection (MMR) study, which allowed us to treat patients with locally advanced cervical cancer surgically [19]. This enabled us to compare clinical staging and MRI using post-operative histology as a reference for cervical cancer up to stage IVA. The aim of this retrospective analysis was to compare the sensitivity, specificity, and predictive values for detection of PMI between MRI and clinical examination under anesthesia (EUA) in cervical cancer stages IB1-IVA, and to determine if factors, such as tumor size or body mass index (BMI), influence test performance of either modality.

\section{Materials and Methods}

\subsection{Patient Selection}

For this retrospective analysis, we identified patients with cervical cancer staged IB to IVA (according to the 2009 FIGO staging criteria [20]) in our study database, who had undergone primary surgical treatment at the University Hospital Leipzig between 10/2000 and $07 / 2017$. All patients were participants of the prospective monocentric observational MMR study at our institution which was initiated in September 1999 to evaluate a novel surgical strategy for the treatment of cervical cancer based on the theory of ontogenetic cancer fields. The study was approved by our institutional ethics review board and was registered retrospectively at the German Clinical Trials Registry (DRKS00015171). A detailed description of the trial has been published [19] and is available at https:/ /www.drks.de/ drks_web /navigate.do?navigationId=trial.HTML\&TRIAL_ID=DRKS00015171 (accessed on 12 June 2021). All patients provided informed consent to participate in this study which included the permission to use data for further analysis. Besides the exclusion criteria specified in the MMR study protocol, such as previous major pelvic surgery and the presence of severe systemic disease prohibiting surgery (American Society of Anesthesiologists [ASA] score $\geq 3$ ), for this current analysis we also excluded women who had undergone neoadjuvant treatment with chemotherapy and patients for whom no MRI-reports were available for review. Therefore, all patients included in this study had undergone preopera- 
tive MRI and had been submitted to EUA. In addition, patients had received cystoscopy and rectoscopy when deemed appropriate by the examiner. Importantly, MR imaging was performed before EUA and the images were displayed in the operating room (augmented EUA, aEUA). The radiology reports (i.e., the written interpretation of the MR images by a board-certified radiologist) were not available during clinical assessment. Throughout this text, aEUA refers to clinical assessment under general anesthesia with synchronous display of the MR images. Of importance, aEUA was performed as a separate procedure before definitive surgical treatment and aEUA does not refer to intra-operative parametrial assessment during radical hysterectomy. The diagnostic information relevant for this current analysis (i.e., data from MRI and aEUA) were gathered retrospectively from our written records. We compared MRI and clinical findings specifically focusing on parametrial involvement. The results were then compared with the pathology reports, which were set as the reference standard. Factors which might influence the accuracy of parametrial assessment were analyzed, such as a patient's body mass index (BMI) and tumor size. In addition, we investigated whether the sensitivity and specificity of parametrial assessment by one examination method could be improved by case stratification according to the test results of the other examination method. For example, we investigated whether detection of parametrial infiltration by MRI was more reliable in the subgroup of patients with parametrial involvement found on aEUA.

\subsection{Statistical Analysis}

Details on statistical analysis can be found in the Supplementary Appendix A. In brief, continuous data are presented in medians and inter-quartile ranges (IQR) while categorical data are given as percentages. Confidence intervals (CI), when applicable, are given for the $95 \%$ range. The sensitivity and specificity for the detection of parametrial tumor invasion of each method of examination, as well as negative predictive value (NPV) and positive predictive value (PPV) were calculated. Accuracy of both MRI and aEUA was compared using the exact McNemar's test. To determine whether the differences in test performance between MRI and aEUA were significant, we applied McNemar's test for paired data to calculate $\chi^{2}$ and $p$-values. To determine the statistical significance of the differences in PPV and NPV for the paired samples, we used the relative predictive value function as proposed by Moskowitz and Pepe. Fisher's exact test was used to calculate statistical significance of differences in sensitivity, specificity, PPV, and NPV between different groups of patients assessed with the same diagnostic method (i.e., unpaired samples, e.g., patients with tumors $<2.5 \mathrm{~cm}$ compared to patients with tumors $\geq 2.5 \mathrm{~cm}$ assessed for parametrial infiltration by MRI). By convention, the differences in the outcome of both methods were considered statistically significant with a $p$-value of 0.05 or less. To ascertain the relevance of potentially influencing factors (BMI, tumor size, parametrial status as assessed by the other examination method) on the accuracy of aEUA and MRI, we built univariable logistic regression models. In addition, we computed a multivariable regression model including all parameters from the univariable regression.

\section{Results}

\subsection{Patient characteristics}

During the study period from 10/2000-05/2017, 551 patients were treated surgically for primary cervical cancer, of which 400 met the inclusion criteria of our study (see Figure 1). A summary of the clinicopathological characteristics of all 400 patients is given in Table 1 (pathology data is from analysis of the post-operative specimen).

The median time interval between acquisition of MR images and aEUA was 1 day (IQR 0-1, range 0-49). In total, 349 patients (87.3\%) had MR images taken on the day of aEUA or the day before. In 5 cases $(1.3 \%)$, the time elapsed between MRI and aEUA was more than 10 days. In all these cases, MRI and aEUA findings regarding parametrial assessment did not differ. 
Parametrial tumor invasion (stage $\geq$ IIB) was detected in 39.3\% clinically, in 39.3\% radiologically, and in $41.3 \%$ pathologically.

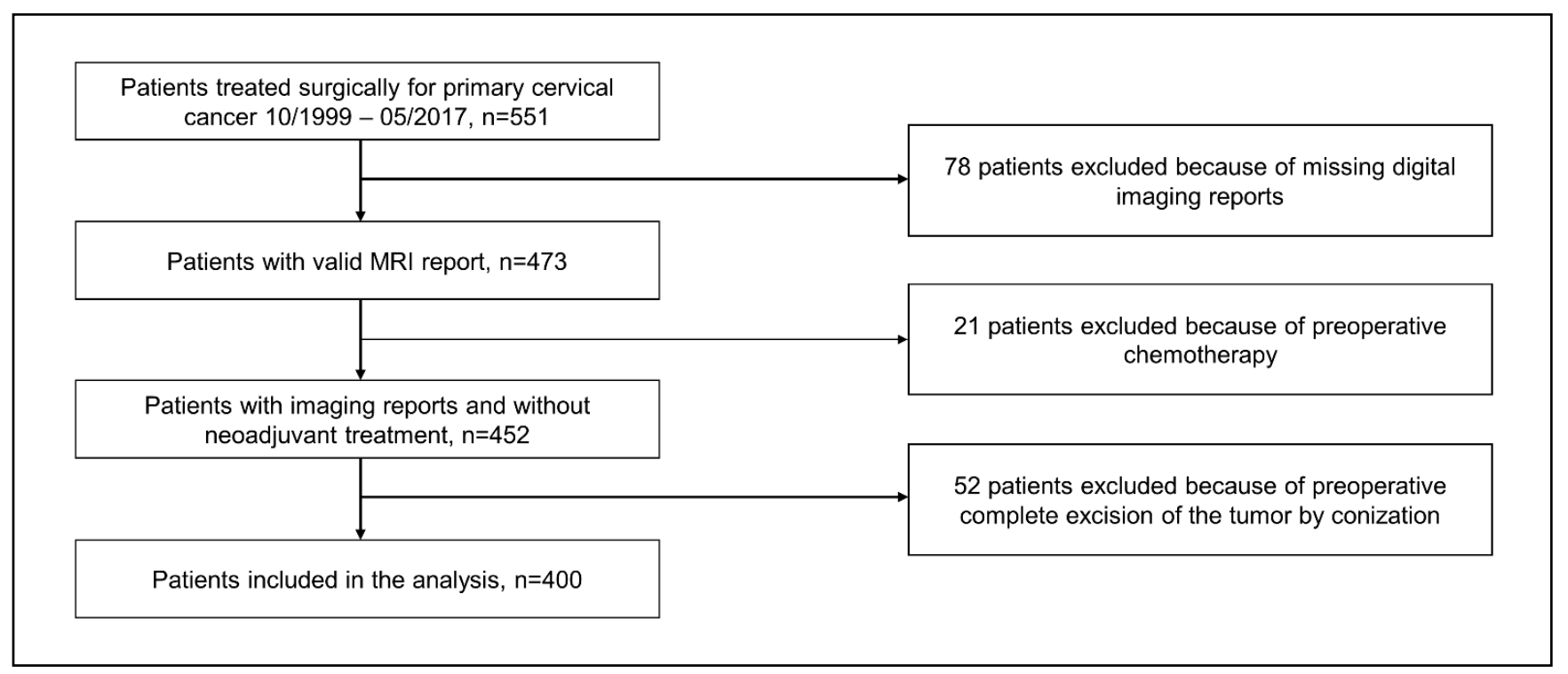

Figure 1. Flowchart depicting the patient selection process.

Table 1. Patient and tumor characteristics.

\begin{tabular}{|c|c|c|c|}
\hline \multicolumn{2}{|c|}{ Parameter } & $n$ & $\%$ \\
\hline \multicolumn{2}{|c|}{ Number of patients } & 400 & 100 \\
\hline \multicolumn{2}{|c|}{ Median age, in years (IQR) } & 46 & $37-55.5$ \\
\hline \multicolumn{2}{|c|}{ Median BMI, in kg/m² (IQR) } & 23 & $21-27$ \\
\hline \multirow{5}{*}{ Histological type } & Squamous cell carcinoma & 302 & 75.5 \\
\hline & Adenocarcinoma & 75 & 18.75 \\
\hline & Adenosquamous carcinoma & 19 & 4.75 \\
\hline & Neuroendocrine carcinoma & 2 & 0.5 \\
\hline & Other & 2 & 0.5 \\
\hline \multirow{8}{*}{ FIGO stage } & IB1 & 174 & 43.5 \\
\hline & IB2 & 36 & 9 \\
\hline & IIA1 & 16 & 4 \\
\hline & IIA2 & 17 & 4.25 \\
\hline & IIB & 136 & 34 \\
\hline & IIIA & 2 & 0.5 \\
\hline & IIIB & 12 & 3 \\
\hline & IVA & 7 & 1.75 \\
\hline \multicolumn{2}{|c|}{ Median tumor size, cm (IQR) } & 3.7 & $2.7-4.9$ \\
\hline \multirow{8}{*}{ pT-stage } & pT1a & 1 & 0.25 \\
\hline & pT1b1 & 159 & 39.75 \\
\hline & pT1b2 & 56 & 14 \\
\hline & pT2a1 & 12 & 3 \\
\hline & pT2a2 & 7 & 1.75 \\
\hline & pT2b & 155 & 38.75 \\
\hline & $\mathrm{pT} 3 \mathrm{~b}$ & 2 & 0.5 \\
\hline & pT4 & 8 & 2 \\
\hline \multirow{5}{*}{ MRI stage (cT) } & сT0 & 51 & 12.75 \\
\hline & cT1b1 & 123 & 30.75 \\
\hline & cT1b2 & 17 & 4.25 \\
\hline & $\mathrm{cT} 2 \mathrm{a}$ & 52 & 13 \\
\hline & $\mathrm{cT} 2 \mathrm{~b}$ & 138 & 34.5 \\
\hline
\end{tabular}


Table 1. Cont.

\begin{tabular}{cccc}
\hline Parameter & & $n$ & $\%$ \\
& cT3a & 2 & 0.5 \\
& cT3b & 1 & 0.25 \\
& cT4 & 16 & 4 \\
Grading & G1 & 58 & 14.5 \\
& G2 & 187 & 46.75 \\
& G3 & 150 & 37.5 \\
& G4 & 1 & 0.25 \\
Lymphovascular involvement & Unknown & 4 & 1 \\
& Yes & 276 & 69 \\
\hline \multirow{2}{*}{ Blood vessel involvement } & Unknown & 121 & 30.25 \\
& Yes & 3 & 0.75 \\
\hline \multirow{2}{*}{ Pelvic lymph node metastasis } & No & 62 & 15.5 \\
\hline Paraaortic lymph node & Unknown & 336 & 84 \\
metastasis & pN1 & 2 & 0.5 \\
\hline
\end{tabular}

BMI: body mass index. FIGO: Fédération Internationale de Gynécologie et d'Obstétrique. IQR: Interquartile range. MRI: magnetic resonance imaging.

\subsection{Assessment for Parametrial Involvement by aEUA Versus MRI}

The distribution of positive and negative test results within our study population is depicted in Figure 2. aEUA exhibited a higher accuracy (83\%) as compared to MRI (76\%). This difference was statistically significant (McNemar's OR $=2.0,95 \%$ CI 1.25-3.27, $p=0.003$ ). Further test results for sensitivity, specificity, and negative and positive predictive values are provided in Table 2 . In summary, aEUA showed statistically significant higher sensitivity, specificity, PPV, and NPV regarding tumor involvement of the parametrium compared to MRI alone.

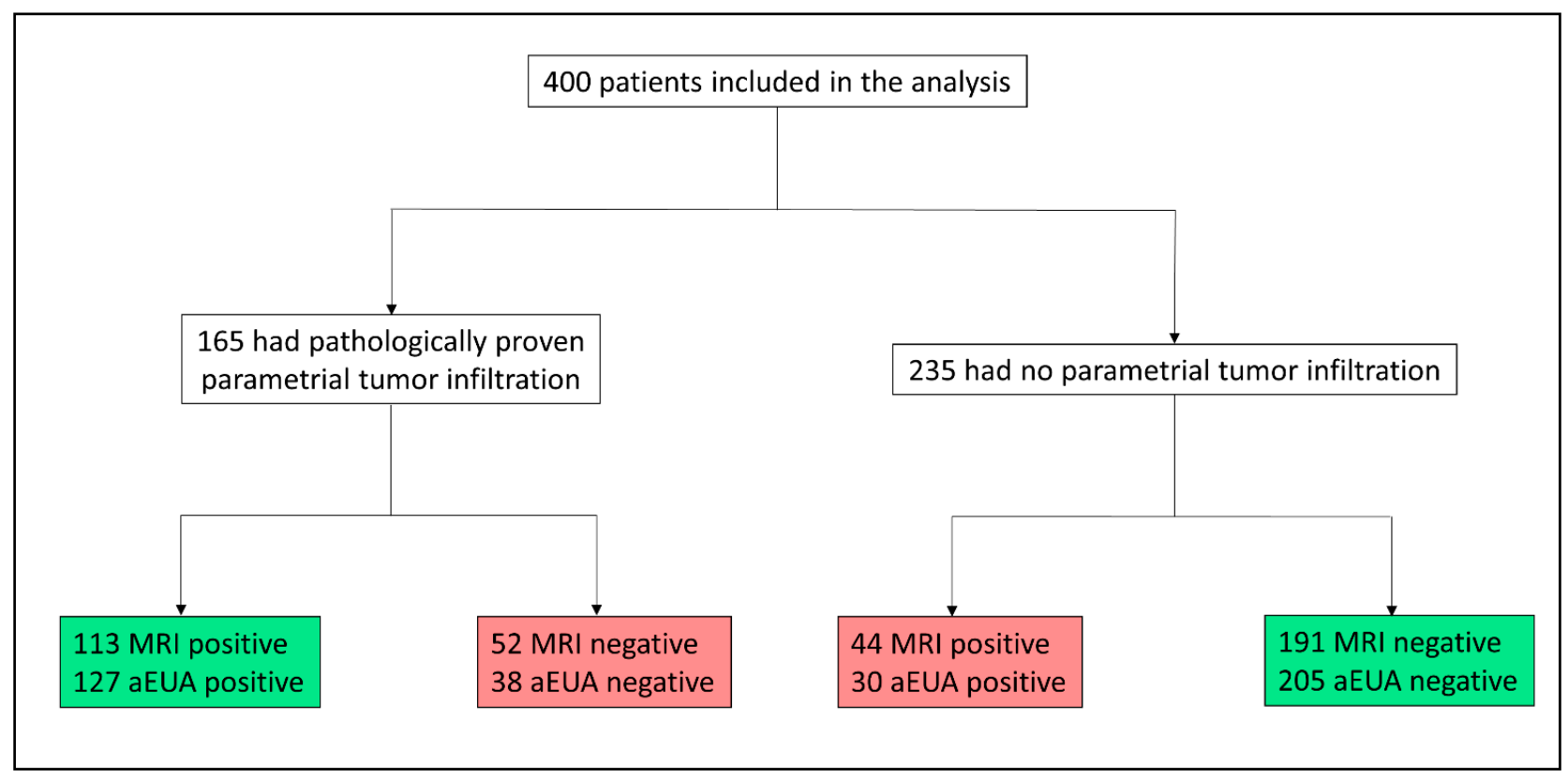

Figure 2. Population tree demonstrating the diagnostic results (MRI and aEUA) within our study population. All accuracy calculations are based on these numbers. aEUA: clinical examination under general anesthesia with display of MR images in the operating room. MRI: magnetic resonance imaging. 
Table 2. Diagnostic performance metrics of MRI and aEUA for pathologically confirmed parametrial infiltration.

\begin{tabular}{|c|c|c|c|c|c|c|c|c|c|c|}
\hline \multirow[b]{2}{*}{ Measure } & \multicolumn{3}{|c|}{ MRI } & \multicolumn{3}{|c|}{ aEUA } & \multicolumn{4}{|c|}{ OR } \\
\hline & Estimate & $\begin{array}{c}\text { Lower } \\
95 \% \text { CI }\end{array}$ & $\begin{array}{c}\text { Upper } \\
95 \% \text { CI }\end{array}$ & Estimate & $\begin{array}{c}\text { Lower } \\
95 \%-C I\end{array}$ & $\begin{array}{c}\text { Upper } \\
95 \% \text { CI }\end{array}$ & Estimate & $\begin{array}{c}\text { Lower } \\
95 \%-C I\end{array}$ & $\begin{array}{c}\text { Upper } \\
95 \%-C I\end{array}$ & $p$-Value \\
\hline Sensitivity & 68.5 & 61.4 & 75.6 & 77.0 & 70.5 & 83.4 & $1.93 *$ & 1.01 & 3.88 & 0.048 * \\
\hline Specificity & 81.4 & 76.3 & 86.3 & 87.2 & 83.0 & 91.5 & $2.08 *$ & 1.04 & 4.38 & 0.038 * \\
\hline PPV & 72.0 & 64.9 & 79.0 & 80.9 & 74.7 & 87.0 & 1.12 ** & 1.03 & 1.22 & $0.007^{* *}$ \\
\hline NPV & 78.6 & 73.4 & 83.8 & 84.4 & 79.8 & 88.9 & $1.07 * *$ & 1.02 & 1.13 & 0.012 ** \\
\hline Accuracy & 76.0 & 71.5 & 80.1 & 83.0 & 79.0 & 86.6 & 2.0 & 1.25 & 3.27 & 0.003 * \\
\hline
\end{tabular}

${ }^{*}$ McNemar's OR, ${ }^{* *}$ Relative predictive value, MRI: magnetic resonance imaging. aEUA: examination under anesthesia with display of MR images (augmented EUA). CI: confidence interval. NPV: negative predictive value. OR: odds ratio. PPV: positive predictive value.

\subsection{Tumor Size as an Influencing Factor on Test Performance}

For this analysis we used tumor sizes as determined by MRI and we used $2.5 \mathrm{~cm}$ maximal tumor diameter as a cut-off value. The cut-off value was chosen based on findings from Woo et al. [21]. Exact tumor size as determined by MRI was available and explicitly stated in the radiology reports of $297(74.3 \%)$ patients. Of these cases, $77(25.9 \%)$ had tumors $<2.5 \mathrm{~cm}$ in diameter, and in $220(74.1 \%)$ tumors measured $\geq 2.5 \mathrm{~cm}$. In the subgroup of patients with tumors $<2.5 \mathrm{~cm}$, pathologically proven parametrial infiltration was present in $10(30 \%)$ of patients. In the subgroup comprising patients with tumors $\geq 2.5$, the prevalence of parametrial infiltration was $61.4 \%$. In summary, specificity and NPV of parametrial assessment by MRI were significantly better in smaller tumors. In aEUA, PPV was better in larger and NPV in smaller tumors (Tables 3 and 4). Although accuracy was not affected by tumor size in aEUA, MRI was associated with a significant drop in accuracy in tumors $\geq 2.5 \mathrm{~cm}$ (univariable logistic regression, OR for a correct diagnosis compared to smaller tumors $0.22, p<0.001)$. This association remained significant in a multivariable regression model (Table 5).

Table 3. Factors influencing the diagnostic performance of MRI in detecting pathologically confirmed parametrial tumor involvement.

\begin{tabular}{|c|c|c|c|c|c|c|c|c|c|c|}
\hline \multirow[b]{2}{*}{ Tumor Size (MRI) } & \multicolumn{3}{|c|}{$<2.5 \mathrm{~cm}(n=77)$} & \multicolumn{3}{|c|}{$>2.5 \mathrm{~cm}(n=220)$} & \multicolumn{3}{|c|}{ OR } & \multirow[b]{2}{*}{$p$-Value } \\
\hline & Estimate & $\begin{array}{l}\text { Lower } \\
95 \% C I\end{array}$ & $\begin{array}{l}\text { Upper } \\
95 \% C I\end{array}$ & Estimate & $\begin{array}{l}\text { Lower } \\
95 \% C I\end{array}$ & $\begin{array}{l}\text { Upper } \\
95 \% C I\end{array}$ & Estimate & $\begin{array}{l}\text { Lower } \\
95 \% \text { CI }\end{array}$ & $\begin{array}{l}\text { Upper } \\
95 \% C I\end{array}$ & \\
\hline Sensitivity & 60 & 29.64 & 90.36 & 70.37 & 62.67 & 78.07 & 1.59 & 0.31 & 7.14 & 0.4917 \\
\hline Specificity & 92.54 & 86.24 & 98.83 & 58.82 & 48.36 & 69.29 & 0.12 & 0.03 & 0.33 & $<0.0001$ \\
\hline PPV & 54.55 & 25.12 & 83.97 & 73.08 & 65.45 & 80.7 & 2.22 & 0.5 & 10.0 & 0.29 \\
\hline NPV & 93.94 & 88.18 & 99.7 & 55.56 & 45.29 & 65.82 & 0.08 & 0.02 & 0.25 & $<0.0001$ \\
\hline Accuracy & 89.47 & 80.31 & 95.34 & 65.91 & 59.24 & 72.15 & 0.26 & 0.11 & 0.55 & $<0.001$ \\
\hline \multirow[b]{2}{*}{ BMI } & \multicolumn{3}{|c|}{$<30 \mathrm{~kg} / \mathrm{m}^{2}(n=337)$} & \multicolumn{3}{|c|}{$\geq 30 \mathrm{~kg} / \mathrm{m}^{2}(n=55)$} & \multicolumn{3}{|c|}{ OR } & \\
\hline & Estimate & $\begin{array}{l}\text { Lower } \\
95 \% \text { CI }\end{array}$ & $\begin{array}{l}\text { Upper } \\
95 \% C I\end{array}$ & Estimate & $\begin{array}{l}\text { Lower } \\
95 \% \mathrm{CI}\end{array}$ & $\begin{array}{l}\text { Upper } \\
95 \% \text { CI }\end{array}$ & Estimate & $\begin{array}{l}\text { Lower } \\
95 \% C I\end{array}$ & $\begin{array}{l}\text { Upper } \\
95 \% C I\end{array}$ & $p$-Value \\
\hline Sensitivity & 66.9 & 59.16 & 74.64 & 80.95 & 64.16 & 97.75 & 2.08 & 0.63 & 9.09 & 0.2201 \\
\hline Specificity & 81.01 & 75.52 & 86.53 & 79.41 & 65.82 & 93.0 & 0.90 & 0.35 & 2.65 & 0.8154 \\
\hline PPV & 71.97 & 64.31 & 79.63 & 70.83 & 52.65 & 89.02 & 0.94 & 0.34 & 2.96 & 1.0 \\
\hline NPV & 77.07 & 71.32 & 82.83 & 87.1 & 75.3 & 99.0 & 2 & 0.65 & 8.27 & 0.25 \\
\hline Accuracy & 75.07 & 70.10 & 79.60 & 80.0 & 67.03 & 89.57 & 1.33 & 0.64 & 2.99 & 0.4997 \\
\hline \multirow[b]{2}{*}{$\begin{array}{c}\text { Clinical Parametrial } \\
\text { Status }\end{array}$} & \multicolumn{3}{|c|}{ Negative $(n=243)$} & \multicolumn{3}{|c|}{ Positive $(n=157)$} & \multicolumn{3}{|c|}{ OR } & \\
\hline & Estimate & $\begin{array}{l}\text { Lower } \\
95 \% C I\end{array}$ & $\begin{array}{l}\text { Upper } \\
95 \% C I\end{array}$ & Estimate & $\begin{array}{l}\text { Lower } \\
95 \% \mathrm{CI}\end{array}$ & $\begin{array}{l}\text { Upper } \\
95 \% C I\end{array}$ & Estimate & $\begin{array}{l}\text { Lower } \\
95 \% C I\end{array}$ & $\begin{array}{l}\text { Upper } \\
95 \% \text { CI }\end{array}$ & $p$-Vlaue \\
\hline Sensitivity & 39.47 & 23.93 & 55.01 & 77.17 & 69.86 & 84.47 & 5.0 & 2.22 & 12.5 & $<0.0001$ \\
\hline Specificity & 86.83 & 82.2 & 91.46 & 43.33 & 25.60 & 61.07 & 0.12 & 0.05 & 0.29 & $<0.0001$ \\
\hline PPV & 35.71 & 21.22 & 50.21 & 85.22 & 78.73 & 91.70 & 12.5 & 5.26 & 33.3 & $<0.0001$ \\
\hline NPV & 88.56 & 84.16 & 92.96 & 30.95 & 16.97 & 44.93 & 0.06 & 0.02 & 0.14 & $<0.0001$ \\
\hline Accuracy & 79.42 & 73.79 & 84.33 & 70.70 & 62.92 & 77.68 & 0.63 & 0.38 & 1.03 & 0.054 \\
\hline
\end{tabular}

BMI: body mass index. CI: confidence interval. MRI: magnetic resonance imaging. NPV: negative predictive value. OR: odds ratio. PPV: positive predictive value. Bold: highlight significant values. 
Table 4. Factors influencing diagnostic performance of aEUA in detecting pathologically confirmed parametrial tumor infiltration.

\begin{tabular}{|c|c|c|c|c|c|c|c|c|c|c|}
\hline \multirow[b]{2}{*}{ Tumor Size (MRI) } & \multicolumn{3}{|c|}{$<2.5 \mathrm{~cm}(n=77)$} & \multicolumn{3}{|c|}{$\geq 2.5 \mathrm{~cm}(n=220)$} & \multicolumn{3}{|c|}{ OR } & \multirow[b]{2}{*}{$p$-Value } \\
\hline & Estimate & $\begin{array}{l}\text { Lower } \\
95 \% \text { CI }\end{array}$ & $\begin{array}{l}\text { Upper } \\
95 \% \text { CI }\end{array}$ & Estimate & $\begin{array}{l}\text { Lower } \\
95 \% \text { CI }\end{array}$ & $\begin{array}{l}\text { Upper } \\
95 \% \text { CI }\end{array}$ & Estimate & $\begin{array}{l}\text { Lower } \\
95 \% \text { CI }\end{array}$ & $\begin{array}{l}\text { Upper } \\
95 \% \text { CI }\end{array}$ & \\
\hline Sensitivity & 70.0 & 41.6 & 98.4 & 77.78 & 70.76 & 84.79 & 0.67 & 0.14 & 4.25 & 0.6954 \\
\hline Specificity & 89.56 & 82.23 & 96.88 & 80.0 & 71.5 & 88.5 & 0.47 & 0.15 & 1.29 & 0.1223 \\
\hline PPV & 50.0 & 23.81 & 76.19 & 86.07 & 79.92 & 92.21 & 0.16 & 0.04 & 0.63 & 0.003 \\
\hline NPV & 95.24 & 90.0 & 100 & 69.39 & 60.26 & 78.51 & 0.16 & 0.02 & 0.4 & $<0.0001$ \\
\hline Accuracy & 87.01 & 77.41 & 93.59 & 78.64 & 72.62 & 83.86 & 0.55 & 0.23 & 1.19 & 0.1307 \\
\hline \multirow[b]{2}{*}{ BMI } & \multicolumn{3}{|c|}{$<30 \mathrm{~kg} / \mathrm{m}^{2}(n=243)$} & \multicolumn{3}{|c|}{$\geq 30 \mathrm{~kg} / \mathrm{m}^{2}(n=157)$} & \multicolumn{3}{|c|}{ OR } & \multirow[b]{2}{*}{$p$-Value } \\
\hline & Estimate & $\begin{array}{l}\text { Lower } \\
95 \% C I\end{array}$ & $\begin{array}{l}\text { Upper } \\
95 \% \text { CI }\end{array}$ & Estimate & $\begin{array}{l}\text { Lower } \\
95 \% \mathrm{CI}\end{array}$ & $\begin{array}{l}\text { Upper } \\
95 \% \text { CI }\end{array}$ & Estimate & $\begin{array}{l}\text { Lower } \\
95 \% C I\end{array}$ & $\begin{array}{l}\text { Upper } \\
95 \% \text { CI }\end{array}$ & \\
\hline Sensitivity & 75.35 & 68.26 & 82.44 & 85.71 & 70.75 & 100 & 0.51 & 0.09 & 1.91 & 0.4105 \\
\hline Specificity & 86.67 & 81.9 & 91.44 & 88.24 & 77.41 & 99.07 & 1.15 & 0.36 & 4.87 & 1.0 \\
\hline PPV & 80.45 & 73.71 & 87.19 & 81.82 & 65.7 & 97.94 & 0.91 & 0.21 & 3.11 & 1.0 \\
\hline NPV & 82.84 & 77.67 & 88.02 & 90.91 & 81.1 & 100 & 2.1 & 0.59 & 11.16 & 0.31 \\
\hline Accuracy & 81.90 & 77.37 & 85.86 & 87.27 & 75.52 & 94.73 & 1.51 & 0.64 & 4.17 & 0.4422 \\
\hline \multirow[b]{2}{*}{$\begin{array}{l}\text { MRI Parametrial } \\
\text { Status }\end{array}$} & \multicolumn{3}{|c|}{ Negative $(n=243)$} & \multicolumn{3}{|c|}{ Positive ( $n=157$ ) } & \multicolumn{3}{|c|}{ OR } & \multirow[b]{2}{*}{$p$-Vlaue } \\
\hline & Estimate & $\begin{array}{l}\text { Lower } \\
95 \% C I\end{array}$ & $\begin{array}{l}\text { Upper } \\
95 \% C I\end{array}$ & Estimate & $\begin{array}{l}\text { Lower } \\
95 \% C I\end{array}$ & $\begin{array}{l}\text { Upper } \\
95 \% C I\end{array}$ & Estimate & $\begin{array}{l}\text { Lower } \\
95 \% C I\end{array}$ & $\begin{array}{l}\text { Upper } \\
95 \% C I\end{array}$ & \\
\hline Sensitivity & 55.77 & 42.27 & 69.27 & 86.73 & 80.47 & 92.98 & 0.2 & 0.08 & 0.45 & $<0.0001$ \\
\hline Specificity & 93.19 & 89.62 & 96.77 & 61.36 & 46.98 & 75.75 & 0.12 & 0.05 & 0.29 & $<0.0001$ \\
\hline PPV & 69.05 & 55.07 & 83.03 & 85.22 & 78.73 & 91.70 & 0.39 & 0.16 & 0.98 & 0.04 \\
\hline NPV & 88.56 & 84.16 & 92.96 & 64.29 & 49.79 & 78.78 & 0.23 & 0.1 & 0.55 & 0.0003 \\
\hline Accuracy & 85.19 & 80.08 & 89.40 & 79.62 & 72.46 & 85.62 & 0.68 & 0.39 & 1.20 & 0.1729 \\
\hline
\end{tabular}

BMI: body mass index. AEUA: Examination under anesthesia with display of MR images. CI: confidence interval. MRI: magnetic resonance imaging. NPV: negative predictive value. OR: odds ratio. PPV: positive predictive value. Bold: highlight significant values.

Table 5. Factors influencing accuracy of parametrial assessment by MRI and aEUA: regression modeling.

\begin{tabular}{|c|c|c|c|c|c|c|}
\hline \multicolumn{7}{|c|}{ Univariable Regression Modeling } \\
\hline $\begin{array}{c}\text { MRI } \\
\text { Parameter }\end{array}$ & $n$ & Estimate & Standard error & z-value & OR & $p$ \\
\hline Size $(<2.5 \mathrm{~cm}$ vs. $\geq 2.5 \mathrm{~cm})$ & 296 & -1.501 & 0.3997 & -3.755 & 0.22 & 0.000173 \\
\hline BMI $\left(<30 \mathrm{~kg} / \mathrm{m}^{2}\right.$ vs. $\left.\geq 30 \mathrm{~kg} / \mathrm{m}^{2}\right)$ & 392 & 0.2717 & 0.3601 & 0.755 & 1.31 & 0.45 \\
\hline $\begin{array}{c}\text { Parametrial status (clinical exam, negative vs. positive) } \\
\text { aEUA }\end{array}$ & 400 & 2.0164 & 0.2868 & 7.032 & 7.511 & $<0.0001$ \\
\hline Parameter & $n$ & Estimate & Standard error & z-value & OR & $p$ \\
\hline Size $(<2.5 \mathrm{~cm}$ vs. $\geq 2.5 \mathrm{~cm})$ & 296 & -0.5839 & 0.3771 & -1.548 & 0.56 & 0.122 \\
\hline $\operatorname{BMI}\left(<30 \mathrm{~kg} / \mathrm{m}^{2}\right.$ vs. $\left.\geq 30 \mathrm{~kg} / \mathrm{m}^{2}\right)$ & 392 & 0.2648 & 0.4078 & 0.649 & 1.3 & 0.516 \\
\hline Parametrial status (MRI, negative vs. positive) & 400 & 2.0164 & 0.2868 & 7.032 & 7.511 & $<0.0001$ \\
\hline \multicolumn{7}{|l|}{ Multivariable Regression Modeling } \\
\hline \multicolumn{7}{|l|}{ MRI } \\
\hline Parameter & $n$ & Estimate & Standard error & z-value & OR & $p$ \\
\hline Size $(<2.5 \mathrm{~cm}$ vs. $\geq 2.5 \mathrm{~cm})$ & 296 & -1.4885 & 0.4188 & -3.554 & 0.23 & 0.00038 \\
\hline BMI $\left(<30 \mathrm{~kg} / \mathrm{m}^{2}\right.$ vs. $\left.\geq 30 \mathrm{~kg} / \mathrm{m}^{2}\right)$ & & 0.2475 & 0.3995 & 0.619 & 1.28 & 0.53565 \\
\hline $\begin{array}{c}\text { Parametrial status (clinical exam, negative vs. positive) } \\
\text { aEUA }\end{array}$ & & 1.7899 & 0.3287 & 5.445 & 5.99 & $<0.0001$ \\
\hline Parameter & $n$ & Estimate & Standard error & z-value & OR & $p$ \\
\hline Size $(<2.5 \mathrm{~cm}$ vs. $\geq 2.5 \mathrm{~cm})$ & 296 & -0.07903 & 0.41051 & -0.193 & 0.92 & 0.847 \\
\hline BMI $\left(<30 \mathrm{~kg} / \mathrm{m}^{2}\right.$ vs. $\left.\geq 30 \mathrm{~kg} / \mathrm{m}^{2}\right)$ & & 0.51993 & 0.48890 & 1063 & 1.68 & 0.288 \\
\hline Parametrial status (MRI, negative vs. positive) & & 1.7899 & 0.3287 & 5.445 & 5.99 & $<0.0001$ \\
\hline
\end{tabular}

BMI: body mass index. aEUA: Examination under anesthesia with display of MR images. CI: confidence interval. MRI: magnetic resonance

imaging. NPV: negative predictive value. OR: odds ratio. PPV: positive predictive value. Bold: highlight significant values. 


\subsection{The Influence of BMI on Testing for Parametrial Invasion}

To evaluate the influence of BMI on sensitivity and specificity for the assessment of parametrial involvement, we compared results of $337(84.3 \%)$ patients with a BMI $<30 \mathrm{~kg} / \mathrm{m}^{2}$ to those of $55(13.8 \%)$ patients with a BMI $\geq 30 \mathrm{~kg} / \mathrm{m}^{2}$. In 8 cases (2\%) the patient's BMI was unknown. Median tumor size in women presenting with a BMI $\geq 30 \mathrm{~kg} / \mathrm{m}^{2}$ was $4 \mathrm{~cm}$. Women with a BMI less than $30 \mathrm{~kg} / \mathrm{m}^{2}$ had tumors with a median size of $3.7 \mathrm{~cm}$. Diagnostic performance measures are presented in Tables 3 and 4. Taken together, BMI had no influence on test performance in either clinical or MRI examination. The same was evident for accuracy assessment by univariable and multivariable logistic regression modeling (Table 5).

\subsection{Incremental Value of Performing Both, Clinical and Radiological Assessment}

We found that sensitivity and PPV of MRI increased from $39.5 \%$ and $35.7 \%$, respectively, in cases without clinical evidence of parametrial involvement $(n=243,60.8 \%)$ to $77.2 \%$ and $85.2 \%$ in tumors with clinical evidence of parametrial infiltration $(n=157,39.2 \%$, $p<0.0001$ for both, sensitivity and PPV). In contrast, specificity and NPV were significantly lower in cases with parametrial infiltration suspected on aEUA (Table 3). Likewise, we analyzed whether aEUA results were better in patients with MRI evidence of parametrial infiltration. Sensitivity and PPV of aEUA was higher in patients with tumors radiologically staged $<\mathrm{cT} 2 \mathrm{~b}$, and, accordingly, lower in patients with tumors staged cT2b and higher (Table 4). Specificity and NPV declined from $93.2 \%$ and $88.7 \%$ for stages $<$ cT2b to $61.4 \%$ and $64.3 \%$ for stages $\geq \mathrm{cT} 2 \mathrm{~b}$ ( $p<0.0001$ for both measures).

\subsection{Accuracy and Net-Sensitivity and Specificity with Combined Examination}

Highest diagnostic accuracy measures were observed when there was agreement regarding parametrial involvement among the two assessment methods. In 315 (78.8\%) congruent cases, parametrial involvement was recognized with a sensitivity of $81.7 \%$ and a specificity of $91.3 \%$. Net sensitivity, i.e., the probability of recognizing true parametrial involvement with a positive result in either MRI, aEUA, or both methods, was $86.1 \%$. Net specificity, i.e., the probability of correctly identifying the absence of parametrial involvement when both MRI and clinical assessment were negative, was $92.8 \%$. Logistic regression models demonstrated that accuracy was significantly higher in the subgroup of patients with concordant results (OR of achieving correct results of 7.5 and 6.0 on univariable and multivariable regression modelling, respectively, $p<0.0001$ in both cases, Table 5).

\subsection{Change of Diagnostic Test Performance over Time}

As the study period spanned almost two decades, we assessed whether diagnostic performance of either method changed over time. We arbitrarily defined three time periods, each spanning 5-6 years, and determined whether accuracy changed over time. There was not a significant change in the accuracy of eather method between the time periods $(p=0.06$ for aEUA and $p=0.34$ for MRI, see Figure 3$)$. 


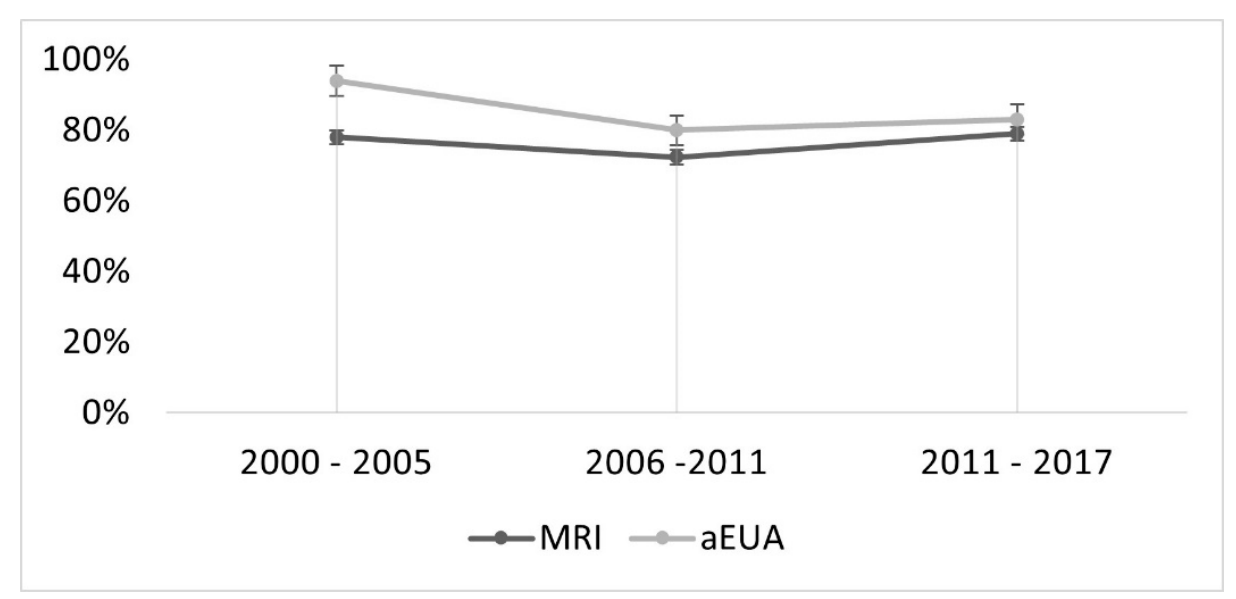

Figure 3. Line graph indicating change in accuracy of MRI and aEUA over time. There was no significant change for both methods ( $p=0.06$ for aEUA and $p=0.34$ for MRI).

\section{Discussion}

This current study was conducted to investigate the accuracy of parametrial assessment in patients with cervical cancer using MRI and clinical examination under general anesthesia. We introduce aEUA, i.e., clinical examination under general anesthesia augmented by display of MR images in the operating room, as a novel concept to improved cervical cancer staging. We found that aEUA results in higher accuracy, sensitivity, specificity, as well as better negative and positive predictive values than MRI alone.

Our findings need to compared to those of other investigators who generally report higher accuracy and better performance of MRI [2,4,8,10,11]. Important limitations to all these studies are, however, that they included only small numbers of patients with tumors staged IIB or higher, and in most cases with IIB disease, no pathological assessment of the parametrium was performed as these patients were generally treated by primary radiotherapy. In one former meta-analysis, pooled sensitivity of PMI assessment in clinical examination was $40 \%$ [4]. However, only four studies with post-operative histopathological assessment from the 1980s, comprising 81 cases in total, included patients with tumors staged IIB or higher [4]. The author's conclusion, that sensitivity of clinical examination was probably underestimated due to a low prevalence of PMI and advanced stages, is undermined by results from our investigation, as we found that sensitivity for clinical examination was $77 \%$. It should be noted, however, that the comparison of our data with that of the aforementioned study is limited by the circumstance that we did not strictly compare clinical examination and MRI, but rather aEUA and MRI alone. The improved sensitivity observed in our study might, therefore, also be a consequence of the different assessment methods.

On the other hand, in our study sensitivity of MRI (68\%) was lower than reported elsewhere, where it ranges up to $100 \%$. Regarding specificity, the difference between aEUA and MRI was less pronounced, though still statistically significant (81.4\% [95\% CI: 76.3-86.3] vs. 87.2\% [95\% CI 83.0-91.5], $p=0.027$ ). Previous studies found comparatively good sensitivity using both examination methods $[4,5,10,14,22]$.

Both specificity and NPV of PMI assessment by MRI were significantly better in smaller tumors $(<2.5 \mathrm{~cm})$. In MRI, parametrial invasion is suspected when disruption of the normal hypointense signal of the cervical stromal ring is present [23], and in tumors with greater size, it is more difficult to distinguish peritumoral edema from actual tumor invasion, leading to an overestimation of tumor stage [24]. Therefore, the higher specificity and NPV in smaller tumors which we observed in our study can be well explained. Because prevalence directly affects predictive values, PPV and NPV are expected to vary with tumor size. In addition, sensitivity and specificity have also been shown to be affected by the prevalence of a given feature (parametrial infiltration) through other mechanisms, though clearly less so than predictive values [25]. Indeed, many studies show a positive correlation 
between tumor size and presence of PMI [26-29], a finding which our data confirms. An important result of our study is, however, that aEUA in contrast to MR imaging alone seems to be less affected by tumor size, as shown by comparison of sensitivity, specificity, and logarithmic regression assessing accuracy (Tables 3-5). This result is best explained by the better performance of clinical palpation in larger tumors, augmented by display of the MR images in the operating room enabling the gynecological oncologist to directly correlate tactile and visual information. Differences in NPV and PPV are plausible considering the higher prevalence of PMI in larger tumors.

There are few studies that investigate the influence of obesity on staging modalities. Although Uppot et al. suggest a lower image quality in obese women [30], there was neither a significant difference in sensitivity and specificity assessing PMI clinically in women with body mass index $\geq 30 \mathrm{~kg} / \mathrm{m}^{2}$ compared to those with BMI $<30 \mathrm{~kg} / \mathrm{m}^{2}$ nor when evaluating PMI with MRI. In fact, there was rather a non-significant increase in sensitivity of PMI detection in both examination methods when patients presented with a BMI over $30 \mathrm{~kg} / \mathrm{m}^{2}$. This observation, however, is likely attributable to a larger median tumor size in this subgroup of patients $(4 \mathrm{~cm})$ which is concordant with our observation that sensitivity is higher in larger tumors.

Best results in sensitivity and specificity for PMI assessment were observed in the subgroup of cases where gynecologists and radiologists agreed on parametrial tumor status, which is consistent with observations made by other investigators $[8,12,31]$. This was also apparent in our univariable and multivariable regression models which demonstrate that accuracy is significantly higher in patients with concordant results. Our results emphasize, therefore, the importance of interdisciplinary case assessment when staging cervical cancer patients.

One aspect of our study regarding the statistical analyses deserves special consideration: We used McNemar's test to calculate OR's to compare accuracy, as well as sensitivity, specificity, and predictive values of MRI and aEUA. In addition, relative predictive values were calculated. For example, considering the accuracy of MRI and aEUA (76\% vs. $83 \%$ ), McNemar's OR was found to be 2.0. Counterintuitively, this does not indicate that aEUA results are twice as often correct as MRI results. Rather, because McNemar's test only considers cases with divergent test results, it means that in cases when MRI and aEUA results are conflicting, aEUA findings will be correct twice as often as MRI findings.

Some limitations of our study should be considered when interpreting these results. First, this study was not a strict comparison of EUA and MR-imaging, as the gynecologic oncologist assessing the patient had access to the MR-images (termed aEUA). This enables the examiner to correlate palpatory findings with pelvic images, thus refining the diagnostic process. At the same time, the radiologists were not routinely informed about clinical findings. Second, our study period spans two decades, and significant progress has been made regarding MRI technology and image analysis. Therefore, our findings might not entirely reflect MRI quality achievable today. Indeed, new algorithms for MR image acquisition and analysis have been established at our institution during the past two years and their effect on diagnostic accuracy will be evaluated prospectively in the near future. However, MRI accuracy did not change significantly over time (Figure 3). Third, it was not a multicentric, prospective study which limits the overall generalizability of our results. Yet, our findings stress the importance of adequate training in attentive, bimanual, rectovaginal palpation of gynecological patients. Last, our study does not provide insight why assessment of larger tumors by MRI is less accurate. It might be related to peritumoral inflammation, but this has to be investigated in future studies.

Significant strengths of our study include that all women underwent surgery and $41 \%$ had pathologically proven parametrial involvement. Furthermore, the number of cases is large compared with most other studies. Moreover, selection bias is minimized because staging did not affect therapy choice.

As this is a retrospective study, prospective evaluation of the aEUA concept is needed. We are currently planning such an investigation at our institution. Further study regarding 
the inferior accuracy of MRI in larger tumors as compared to smaller tumors is also needed. It would be especially interesting to evaluate whether peritumoral inflammation contributes to reduced specificity in larger tumors.

As most women with locally advanced cervical cancer are treated with primary chemoradiotherapy, the majority of patients with suspected parametrial tumor infiltration will not receive surgery irrespective whether parametrial tumor extension is suspected on MRI or aEUA. However, our findings should be kept in mind when interpreting MR images from cervical cancer patients, especially with larger tumors. Irrespective of the treatment pursued, optimal pathoanatomical diagnosis of the disease is mandatory.

\section{Conclusions}

We demonstrate that clinical evaluation of the parametrium under general anesthesia with display of MR images in the operating room, performed by a gynecologic oncologist, is more accurate than MR imaging alone in detecting parametrial tumor involvement by cervical cancer. Display of MR images in the operating room for assessment by the examining gynecologic oncologist should, therefore, be added to standard examination under anesthesia. In addition, our data shows that MRI assessment of smaller tumors $(<2.5 \mathrm{~cm})$ is more reliable compared to larger tumors. BMI does not affect diagnostic accuracy of either clinical or MRI examination. Future prospective studies need to confirm these findings.

Author Contributions: Conceptualization, B.W., L.-C.H., M.H., and B.A.; methodology, B.W., M.H., and B.A.; formal analysis, P.S., M.L., and B.W.; investigation, P.S., M.L., and B.W.; resources, B.A., M.H., M.M., and L.-C.H.; data curation, P.S., M.L., and B.W.; writing-original draft preparation, P.S. and B.W.; writing-review and editing, M.L., M.M., L.-C.H., M.H., and B.A.; visualization, P.S. and B.W.; supervision, B.A. and M.H.; project administration, B.W.; funding acquisition, B.A. and M.H. All authors have read and agreed to the published version of the manuscript.

Funding: The research was in part supported by Stiftung Gynäkologische Onkologie, a non-profit foundation dedicated to the support of research in gynecologic oncology. The foundation had no role in the collection, analysis, and interpretation of data, in the writing of the report, and in the decision to submit the article for publication.

Institutional Review Board Statement: The study was conducted according to the guidelines of the Declaration of Helsinki. The MMR trial, as part of which the data analyzed in this publication were collected, was approved by the Ethics Committee of the University of Leipzig (151/2000, 192/2001, and 012/13-28012013; initial approval was granted on 22 September 2000, and the subsequent amendments were approved on 17 October 2007 and 6 March 2013).

Informed Consent Statement: Informed consent was obtained from all subjects involved in the study.

Data Availability Statement: The data presented in this study are available on request from the corresponding author. The data are not publicly available because it contains sensitive patient information.

Conflicts of Interest: The authors declare that there are no competing interests relevant to this investigation. Outside the submitted work, BA has received honoraria or consulting fees from AstraZeneca, Novartis, Roche, and Pfizer. In addition, BA received compensation for travel and accommodation expenses from Roche, Pfizer, Novartis, Celgene, and Daiichi Sankyo. BW is stockholder of BioNTech AG.

\section{Appendix A. Statistical Analysis in Detail}

All data were recorded in Excel spreadsheets (Microsoft, 2016) and comma-separatedvariable (CSV) tables were created for further statistical processing in $\mathrm{R}$, an open-source statistical software [32]. Continuous data are presented in medians and inter-quartile ranges (IQR) while categorical data are given as percentages. Confidence intervals (CI), when applicable, are given for the $95 \%$ range. 
The sensitivity and specificity for the detection of parametrial tumor invasion of each method of examination, as well as negative predictive value (NPV) and positive predictive value (PPV) were calculated. For this purpose, results from MRI scans and aEUA were compared with the pathological findings in $2 \times 2$ tables. We used the DTComPair package for $\mathrm{R}$ to calculate the confidence intervals for the different test performance measures. Accuracy of both MRI and aEUA was compared by tabulating a contingency table including the number of correctly and incorrectly diagnosed patients for each method and then using the exact McNemar's test [33] included the exact $2 \times 2$ package for $\mathrm{R}$, providing confidence intervals and odds ratios (OR).

To determine whether the differences in test performance between MRI and aEUA were significant, we used contingency tables including either only patients with parametrial infiltration (sensitivity), or without parametrial infiltration (specificity). We then applied McNemar's test for paired data to calculate $\chi^{2}$ and $p$-value [34]. To determine the statistical significance of the differences in PPV and NPV for the paired samples, we used the relative predictive value function as proposed by Moskowitz and Pepe, implanted in the DTComPair package for R [35].

Statistical significance of differences in sensitivity, specificity, and accuracy between different groups of patients assessed with the same diagnostic method (i.e., unpaired samples, e.g., patients with tumors $<2.5 \mathrm{~cm}$ compared to patients with tumors $\geq 2.5 \mathrm{~cm}$ assessed for parametrial infiltration by MRI) was calculated using Fisher's exact test as implemented in R. For calculation of difference in PPV and NPV we used modified contingency tables only including patients with a positive test result (PPV) and with a negative test result (NPV), again using Fisher's exact test.

To ascertain the relevance of potentially influencing factors (BMI, tumor size, parametrial status as assessed by the other examination method) on the accuracy of aEUA and MRI, we built univariable logistic regression models using the glm function implemented in R. In addition, we computed a multivariable regression model including all parameters from the univariable regression.

\section{References}

1. Bhatla, N.; Berek, J.S.; Cuello Fredes, M.; Denny, L.A.; Grenman, S.; Karunaratne, K.; Kehoe, S.T.; Konishi, I.; Olawaiye, A.B.; Prat, J.; et al. Revised FIGO staging for carcinoma of the cervix uteri. Int. J. Gynaecol. Obstet. 2019, 145, 129-135. [CrossRef] [PubMed]

2. Kraljević, Z.; Visković, K.; Ledinsky, M.; Zadravec, D.; Grbavac, I.; Bilandzija, M.; Soljacić-Vranes, H.; Kuna, K.; Klasnić, K.; Krolo, I. Primary uterine cervical cancer: Correlation of preoperative magnetic resonance imaging and clinical staging (FIGO) with histopathology findings. Coll. Antropol. 2013, 37, 561-568.

3. Narayan, K.; Lin, M.Y. Staging for cervix cancer: Role of radiology, surgery and clinical assessment. Best Pract. Res. Clin. Obstet. Gynaecol. 2015, 29, 833-844. [CrossRef] [PubMed]

4. Thomeer, M.G.; Gerestein, C.; Spronk, S.; van Doorn, H.C.; van der Ham, E.; Hunink, M.G. Clinical examination versus magnetic resonance imaging in the pretreatment staging of cervical carcinoma: Systematic review and meta-analysis. Eur. Radiol. 2013, 23, 2005-2018. [CrossRef] [PubMed]

5. Woo, S.; Suh, C.H.; Kim, S.Y.; Cho, J.Y.; Kim, S.H. Magnetic resonance imaging for detection of parametrial invasion in cervical cancer: An updated systematic review and meta-analysis of the literature between 2012 and 2016. Eur. Radiol. 2018, 28, 530-541. [CrossRef]

6. Ozsarlak, O.; Tjalma, W.; Schepens, E.; Corthouts, B.; Beeck, B.O.d.; van Marck, E.; Parizel, P.M.; de Schepper, A.M. The correlation of preoperative CT, MR imaging, and clinical staging (FIGO) with histopathology findings in primary cervical carcinoma. Eur. Radiol. 2003, 13, 2338-2345. [CrossRef]

7. Bipat, S.; Glas, A.S.; van der Velden, J.; Zwinderman, A.H.; Bossuyt, P.M.M.; Stoker, J. Computed tomography and magnetic resonance imaging in staging of uterine cervical carcinoma: A systematic review. Gynecol. Oncol. 2003, 91, 59-66. [CrossRef]

8. Hricak, H.; Gatsonis, C.; Chi, D.S.; Amendola, M.A.; Brandt, K.; Schwartz, L.H.; Koelliker, S.; Siegelman, E.S.; Brown, J.J.; McGhee, R.B.; et al. Role of imaging in pretreatment evaluation of early invasive cervical cancer: Results of the intergroup study American College of Radiology Imaging Network 6651-Gynecologic Oncology Group 183. J. Clin. Oncol. 2005, 23, 9329-9337. [CrossRef]

9. Hancke, K.; Heilmann, V.; Straka, P.; Kreienberg, R.; Kurzeder, C. Pretreatment staging of cervical cancer: Is imaging better than palpation?: Role of CT and MRI in preoperative staging of cervical cancer: Single institution results for 255 patients. Ann. Surg. Oncol. 2008, 15, 2856-2861. [CrossRef]

10. Chung, H.H.; Kang, S.-B.; Cho, J.Y.; Kim, J.W.; Park, N.-H.; Song, Y.-S.; Kim, S.H.; Lee, H.-P. Can preoperative MRI accurately evaluate nodal and parametrial invasion in early stage cervical cancer? Jpn. J. Clin. Oncol. 2007, 37, 370-375. [CrossRef] 
11. Qin, Y.; Peng, Z.; Lou, J.; Liu, H.; Deng, F.; Zheng, Y. Discrepancies between clinical staging and pathological findings of operable cervical carcinoma with stage IB-IIB: A retrospective analysis of 818 patients. Aust. N. Z. J. Obstet. Gynaecol. 2009, $49,542-544$. [CrossRef]

12. Zhang, W.; Chen, C.; Liu, P.; Li, W.; Hao, M.; Zhao, W.; Lu, A.; Ni, Y. Impact of pelvic MRI in routine clinical practice on staging of IB1-IIA2 cervical cancer. Cancer Manag. Res. 2019, 11, 3603-3609. [CrossRef] [PubMed]

13. Qu, J.-R.; Qin, L.; Li, X.; Luo, J.-P.; Li, J.; Zhang, H.-K.; Wang, L.; Shao, N.-N.; Zhang, S.-N.; Li, Y.-L.; et al. Predicting Parametrial Invasion in Cervical Carcinoma (Stages IB1, IB2, and IIA): Diagnostic Accuracy of T2-Weighted Imaging Combined with DWI at 3 T. AJR Am. J. Roentgenol. 2018, 210, 677-684. [CrossRef]

14. Shweel, M.A.; Abdel-Gawad, E.A.; Abdel-Gawad, E.A.; Abdelghany, H.S.; Abdel-Rahman, A.M.; Ibrahim, E.M. Uterine Cervical Malignancy: Diagnostic Accuracy of MRI with Histopathologic Correlation. J. Clin. Imaging Sci. 2012, 2. [CrossRef] [PubMed]

15. Marth, C.; Landoni, F.; Mahner, S.; McCormack, M.; Gonzalez-Martin, A.; Colombo, N. Cervical cancer: ESMO Clinical Practice Guidelines for diagnosis, treatment and follow-up. Ann. Oncol. 2017, 28, iv72-iv83. [CrossRef]

16. Koh, W.-J.; Abu-Rustum, N.R.; Bean, S.; Bradley, K.; Campos, S.M.; Cho, K.R.; Chon, H.S.; Chu, C.; Clark, R.; Cohn, D.; et al. Cervical Cancer, Version 3.2019, NCCN Clinical Practice Guidelines in Oncology. J. Natl. Compr. Cancer Netw. 2019, 17, 64-84. [CrossRef]

17. Yang, K.; Park, W.; Huh, S.J.; Park, B.K.; Kim, C.K.; Kim, B.-G.; Bae, D.-S.; Lee, J.-W. Parametrial Involvement on Magnetic Resonance Imaging Has No Effect on the Survival of Early-Stage Cervical Cancer Patients. Int. J. Gynecol. Cancer 2017, 27, 507-513. [CrossRef] [PubMed]

18. Zreik, T.G.; Chambers, J.T.; Chambers, S.K. Parametrial involvement, regardless of nodal status: A poor prognostic factor for cervical cancer. Obstet. Gynecol. 1996, 87, 741-746. [CrossRef]

19. Höckel, M.; Wolf, B.; Schmidt, K.; Mende, M.; Aktas, B.; Kimmig, R.; Dornhöfer, N.; Horn, L.-C. Surgical resection based on ontogenetic cancer field theory for cervical cancer: Mature results from a single-centre, prospective, observational, cohort study. Lancet Oncol. 2019, 20, 1316-1326. [CrossRef]

20. Pecorelli, S. Revised FIGO staging for carcinoma of the vulva, cervix, and endometrium. Int. J. Gynaecol. Obstet. 2009, 105, 103-104. [CrossRef]

21. Woo, S.; Moon, M.H.; Cho, J.Y.; Kim, S.H.; Kim, S.Y. Diagnostic Performance of MRI for Assessing Parametrial Invasion in Cervical Cancer: A Head-to-Head Comparison between Oblique and True Axial T2-Weighted Images. Korean J. Radiol. 2019, 20, 378-384. [CrossRef] [PubMed]

22. Dappa, E.; Elger, T.; Hasenburg, A.; Düber, C.; Battista, M.J.; Hötker, A.M. The value of advanced MRI techniques in the assessment of cervical cancer: A review. Insights Imaging 2017, 8, 471-481. [CrossRef]

23. Balcacer, P.; Shergill, A.; Litkouhi, B. MRI of cervical cancer with a surgical perspective: Staging, prognostic implications and pitfalls. Abdom. Radiol. 2019, 44, 2557-2571. [CrossRef] [PubMed]

24. Kaur, H.; Silverman, P.M.; Iyer, R.B.; Verschraegen, C.F.; Eifel, P.J.; Charnsangavej, C. Diagnosis, staging, and surveillance of cervical carcinoma. AJR Am. J. Roentgenol. 2003, 180, 1621-1631. [CrossRef]

25. Leeflang, M.M.G.; Rutjes, A.W.S.; Reitsma, J.B.; Hooft, L.; Bossuyt, P.M.M. Variation of a test's sensitivity and specificity with disease prevalence. CMAJ 2013, 185, E537-E544. [CrossRef] [PubMed]

26. Kim, M.; Suh, D.H.; Kim, K.; Lee, H.J.; Kim, Y.B.; No, J.H. Magnetic Resonance Imaging as a Valuable Tool for Predicting Parametrial Invasion in Stage IB1 to IIA2 Cervical Cancer. Int. J. Gynecol. Cancer 2017, 27, 332-338. [CrossRef]

27. Canaz, E.; Ozyurek, E.S.; Erdem, B.; Aldikactioglu Talmac, M.; Yildiz Ozaydin, I.; Akbayir, O.; Numanoglu, C.; Ulker, V. Preoperatively Assessable Clinical and Pathological Risk Factors for Parametrial Involvement in Surgically Treated FIGO Stage IB-IIA Cervical Cancer. Int. J. Gynecol. Cancer 2017, 27, 1722-1728. [CrossRef]

28. Frumovitz, M.; Sun, C.C.; Schmeler, K.M.; Deavers, M.T.; Dos Reis, R.; Levenback, C.F.; Ramirez, P.T. Parametrial involvement in radical hysterectomy specimens for women with early-stage cervical cancer. Obstet. Gynecol. 2009, 114, 93-99. [CrossRef]

29. He, F.; Du, J.; Chen, X.; He, L. Assessment of Parametrial Involvement in Early Stages Cervical Cancer with Preoperative Magnetic Resonance Imaging. Int. J. Gynecol. Cancer 2018, 28, 1758-1765. [CrossRef]

30. Uppot, R.N.; Sahani, D.V.; Hahn, P.F.; Kalra, M.K.; Saini, S.S.; Mueller, P.R. Effect of obesity on image quality: Fifteen-year longitudinal study for evaluation of dictated radiology reports. Radiology 2006, 240, 435-439. [CrossRef]

31. Bourgioti, C.; Chatoupis, K.; Rodolakis, A.; Antoniou, A.; Tzavara, C.; Koutoulidis, V.; Moulopoulos, L.A. Incremental prognostic value of MRI in the staging of early cervical cancer: A prospective study and review of the literature. Clin. Imaging 2016, 40, 72-78. [CrossRef]

32. R Core Team. R: A Language and Environment for Statistical; R Foundation for Statistical Computing: Vienna, Austria, 2019; Available online: https:/ / www.R-project.org/ (accessed on 12 June 2021).

33. McNemar, Q. Note on the sampling error of the difference between correlated proportions or percentages. Psychometrika 1947, 12, 153-157. [CrossRef] [PubMed]

34. Kim, S.; Lee, W. Does McNemar's test compare the sensitivities and specificities of two diagnostic tests? Stat. Methods Med. Res. 2017, 26, 142-154. [CrossRef] [PubMed]

35. Moskowitz, C.S.; Pepe, M.S. Comparing the predictive values of diagnostic tests: Sample size and analysis for paired study designs. Clin. Trials 2006, 3, 272-279. [CrossRef] [PubMed] 